\title{
Postoperative decrease of albumin ( $\Delta$ Alb) as early predictor of complications after gastrointestinal surgery: a systematic review
}

\author{
Gaëtan-Romain Joliat ${ }^{1,2^{*}}$ (D, Arnaud Schoor ${ }^{3}$, Markus Schäfer ${ }^{1}$, Nicolas Demartines ${ }^{1}$, Martin Hübner ${ }^{1}$ and \\ Ismail Labgaa'
}

\begin{abstract}
Background: Postoperative complications are frequent after gastrointestinal surgery and early prediction remains an unmet need. Serum albumin shows a rapid decrease after surgery, and this decline $(\Delta \mathrm{Alb})$ may reflect the intensity of the surgical stress response and thereby be a predictor of postoperative complications. This study aimed to comprehensively review the available data on $\Delta \mathrm{Alb}$ in gastrointestinal surgery.

Methods: PRISMA guidelines were followed to conduct a systematic review of the literature in MEDLINE and Embase. Studies assessing the role of $\Delta$ Alb to predict complications after gastrointestinal surgery were included.

Results: A total of 1256 articles were screened, and 16 studies were included in the final analysis: 7 prospective and 9 retrospective trials. Sensitivity of $\Delta$ Alb to predict postoperative complications ranged from 63 to $84 \%$, whereas specificity ranged from 61 to $86 \%$. Nine out of the 16 included studies established a threshold of $\Delta$ Alb to predict morbidity (range: $5-11 \mathrm{~g} / \mathrm{l}$ or $14-27 \%$ ).
\end{abstract}

Conclusion: $\Delta$ Alb appeared as a valuable and promising biomarker to anticipate complications after gastrointestinal surgery. Future efforts are needed to determine whether and how $\Delta \mathrm{Alb}$ may be integrated in clinical practice to guide clinicians in the perioperative management of patients.

Keywords: Complications, Predictor, $\Delta$ Alb, Biomarkers

\section{Background}

Gastrointestinal (GI) surgery is associated with a substantial risk of postoperative complications, reported in almost $50 \%$ of cases after major operations (Jarnagin et al., 2002). Not only detrimental to patients, they also induce a significant increase in costs, a timely concern while healthcare expenditures particularly need to be

\footnotetext{
* Correspondence: gaetan.joliat@gmail.com

'Department of Visceral Surgery, Lausanne University Hospital (CHUV), University of Lausanne (UNIL), Rue du Bugnon 46, CH-1011 Lausanne, Switzerland

${ }^{2}$ Graduate School of Health Sciences, University of Bern, Bern, Switzerland Full list of author information is available at the end of the article
}

controlled (Straatman et al., 2015; Vonlanthen et al., 2011). It is important for surgeons to identify preoperatively patients at risk (e.g., patients with pre-existing cardiopulmonary pathology or with malnutrition) and to rapidly recognize patients who will develop postoperative complications. Being the first step to anticipate complications, early identification of patients at risk is a sine qua non condition to succeed. To this regard, available biomarkers such as white blood cells count, Creactive protein $(\mathrm{CRP})$, procalcitonin, or interleukin-6 are quite disappointing, being poorly accurate, with low sensitivity or specificity, with slow kinetics, relatively late

(c) The Author(s). 2022 Open Access This article is licensed under a Creative Commons Attribution 4.0 International License, which permits use, sharing, adaptation, distribution and reproduction in any medium or format, as long as you give appropriate credit to the original author(s) and the source, provide a link to the Creative Commons licence, and indicate if changes were made. The images or other third party material in this article are included in the article's Creative Commons licence, unless indicated otherwise in a credit line to the material. If material is not included in the article's Creative Commons licence and your intended use is not permitted by statutory regulation or exceeds the permitted use, you will need to obtain permission directly from the copyright holder. To view a copy of this licence, visit http://creativecommons.org/licenses/by/4.0/ The Creative Commons Public Domain Dedication waiver (http://creativecommons.org/publicdomain/zero/1.0/) applies to the data made available in this article, unless otherwise stated in a credit line to the data. 
indicators, expensive, or difficultly reproducible (Labgaa et al., 2017a). An example of a biomarker that changed the management is the negative predictive value of CRP after colorectal surgery. A meta-analysis showed that discharge based on the value of CRP was safe after colorectal surgery (Warschkow et al., 2012). Another example is the realization of CT scan based on postoperative CRP values. This strategy has been shown in colorectal surgery to detect postoperative complications earlier (McSorley et al., 2017).

Albumin is a negative acute phase protein with fast decline in case of inflammation. This phenomenon is mainly caused by redistribution into the third space and was observed already during the first hours after various types of surgical procedures (Labgaa et al., 2017b). Moreover, the degree of albumin decrease was commensurate with the surgical trauma. In addition, surgical trauma (extent of surgery) was associated with stress response which is also associated with postoperative complications (Thorell et al., 1999). In that context, the amplitude of albumin decrease was referred as $\Delta \mathrm{Alb}$ and recent studies investigated whether it could be used as a predictor of postoperative complications. In addition of displaying attractive characteristics such as being performant, early indicative, easy to measure, and inexpensive, these studies revealed promising data on its predictive value for postoperative complications (Labgaa et al., 2017b). Enhanced Recovery After Surgery (ERAS) is a multimodal perioperative management pathway with the goal of improving postoperative recovery by decreasing the response to surgical stress. ERAS via goal-directed fluid therapy or rapid onset of oral nutrition might have an influence on $\Delta \mathrm{Alb}$ that reflects the magnitude of the inflammatory response to the surgical trauma. In liver surgery, ERAS has been shown to decrease $\triangle \mathrm{Alb}$ compared to non-ERAS patients (median $\Delta$ Alb: 12 vs. $16 \mathrm{~g} / \mathrm{l}$, $p<0.001$ ) (Gonvers et al., 2021).

The present study aimed to perform a systematic review of available data on $\triangle \mathrm{Alb}$ in GI surgery.

\section{Methods}

\section{Search}

A systematic review of the literature was performed using MEDLINE/PubMed and Embase. MeSH terms used were "albumin" and "postoperative complications." Non-MeSH terms (free terms) were "perioperative albumin level," "delta albumin," and "postoperative morbidity." These terms were combined with AND or $\mathrm{OR}$ in the research equation. Previous terms were then adapted for Embase. Cross-referencing of all bibliographies of included articles was also performed. Search results extended from January 1, 1980, to November 30, 2020. Literature search was independently performed by two authors (GRJ, AS). In case of disagreement for study inclusion, the final decision was made by the senior author (IL).

\section{Eligibility criteria}

Inclusion criteria were studies that evaluated $\Delta \mathrm{Alb}$ as predictor of postoperative overall complication or specific complication. $\Delta \mathrm{Alb}$ (in $\mathrm{g} / \mathrm{L}$ or $\%$ ) was defined as the difference between the preoperative serum albumin level and the postoperative serum level. Only articles concerning GI surgery were included. Studies that assessed only preoperative or postoperative albumin were excluded. Albumin ratio or other scores including albumin were not considered. Only full-text studies written in English were considered. Articles regarding transplantation (i.e., kidney, pancreas, and liver) were also considered. All study types except meta-analyses and systematic reviews were eligible.

Included studies were then analyzed and summarized. They were grouped in the results according to the types of GI surgery. If a study included a mix of GI procedures, it was classified as abdominal surgery.

\section{Extraction of data}

Mean or median $\Delta$ Alb levels (in $\mathrm{g} / \mathrm{L}$ ) and percentage of $\Delta$ Alb were extracted from included articles. It was also assessed if a threshold of $\Delta$ Alb was defined as discriminative for complication and if a multivariable regression analysis was performed.

\section{Quality assessment, heterogeneity, and statistics}

The Newcastle-Ottawa Scale was used to evaluate the quality of included studies. As this review assessed the accuracy of a diagnostic test $(\Delta \mathrm{Alb})$, Cochrane $I^{2}$ for heterogeneity or funnel plot could not be applied. Instead, a summary receiver operating characteristic (SROC) graph was generated. The SROC graph plotted all results of sensitivity and specificity of the included studies. Review Manager (RevMan, version 5.3, Copenhagen: The Nordic Cochrane Centre, The Cochrane Collaboration, 2014) was used. The present review followed the PRISMA (Preferred Reporting Items for Systematic Reviews and Meta-Analyses) and AMSTAR (Assessing the methodological quality of systematic reviews) guidelines.

As no patient data were collected, no approval of the institutional review board was needed. The methods of the review were established prior to the conduct of the review (including the review question, search strategy, inclusion/ exclusion criteria, and assessment of risk of bias).

\section{Results}

A total of 1256 articles were screened. Based on titles and abstracts, 1223 articles were excluded, and 33 fulltext articles were assessed for eligibility. Finally, 16 studies were included in this review as shown in the 
flowchart (Fig. 1). Table 1 summarizes these studies. Data are presented hereunder based on the types of surgery. Use of exogenous albumin was not routinely used in all included studies, except in the study by Giovannini et al. (Giovannini et al., 2006).

\section{Abdominal surgery}

In a pilot prospective study with 70 patients undergoing abdominal surgery, postoperative CRP and albumin deltas were found to reflect the magnitude of surgical stress response defined by the inflammatory and metabolic reactions to the surgical trauma (Mantziari et al., 2015; Hübner et al., 2016). Serum albumin was measured preoperatively and on every postoperative day (POD) until POD 5 if the patient was still hospitalized. The maximum $\Delta$ Alb was larger in patients with postoperative complications (mean $\Delta$ Alb: 10 vs. 6.1, $p=0.005)$ and correlated with longer length of stay (LoS, rho $=0.285, p<0.020$ ), blood loss (rho $=0.605$, $p<0.001$ ), surgery duration ( $\mathrm{rho}=0.47, p<0.001$ ), and CRP level (rho=0.391, $p=0.002$ ). These results were confirmed by a larger prospective study from the same group including 138 patients where postoperative albumin values were measured until POD 3 (Labgaa et al., 2017c). $\Delta$ Alb correlated with the occurrence of postoperative complications and a cut-off of $\geq 10 \mathrm{~g} / \mathrm{l}$ was associated with a three-fold risk of developing postoperative complications.

Galata et al. retrospectively reviewed the nutritional and prognostic role of albumin in patients undergoing intestinal surgery (small bowel and colorectal procedures) (Galata et al., 2020). $\Delta$ Alb on postoperative day

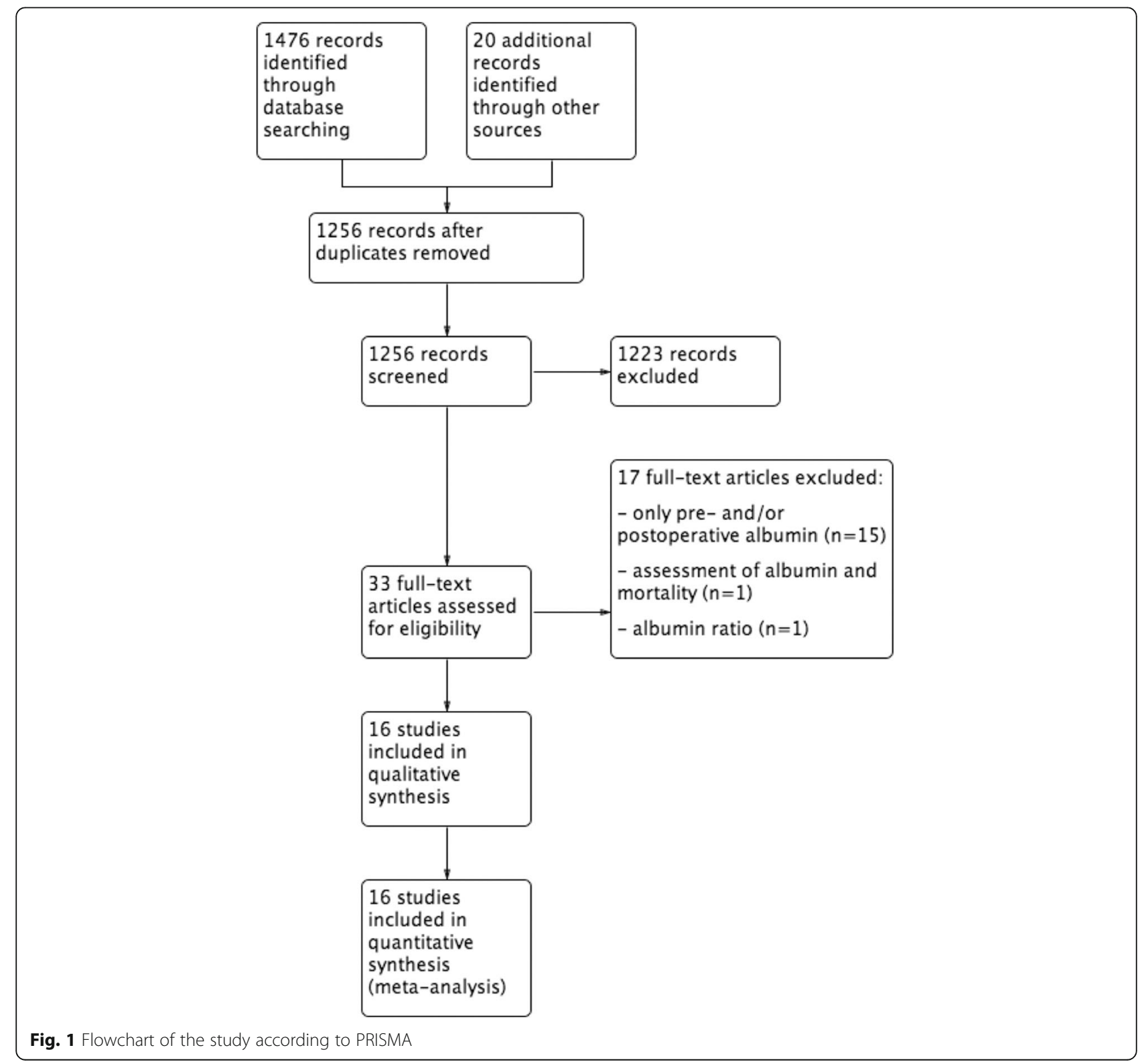


Table 1 Characteristics and main outcomes of all included articles $(n=16)$ assessing the predictive role of $\Delta$ Alb in terms of postoperative complications

\begin{tabular}{|c|c|c|c|c|c|c|c|}
\hline Authors & Year & Design & Surgery & $N$ & Primary outcome & Delta Albumin threshold & MV analysis ${ }^{\mathrm{a}}$ \\
\hline Mantziari et al. & 2015 & Prosp & Abdominal & 70 & Metabolic profile & $\mathrm{N} / \mathrm{A}$ & Yes $^{\mathrm{b}}$ \\
\hline Hübner et al. & 2016 & Prosp & Abdominal & 70 & Stress response and compl & N/A & N/A \\
\hline Labgaa et al. & 2017 & Prosp & Abdominal & 138 & Stress response & $10 \mathrm{~g} / \mathrm{L}$ & Yes \\
\hline Kumar et al. & 2020 & Prosp & Abdominal & 50 & Compl & N/A & N/A \\
\hline Müller et al. & 2018 & Retro & Intestinal & 182 & Compl & $24 \%$ & Yes \\
\hline Galata et al. & 2020 & Retro & Intestinal & 103 & Major compl & $27 \%$ & N/A \\
\hline Fan et al. & 1989 & Prosp & Esophagus & 40 & Compl & N/A & N/A \\
\hline Labgaa et al. & 2020 & Retro & Esophagus & 1046 & Major compl & $11 \mathrm{~g} / \mathrm{L}$ & Yes \\
\hline Liu et al. & 2017 & Retro & Gastric & 223 & Compl. & $14 \%$ & Yes \\
\hline Ai et al. & 2019 & Retro & Gastric & 193 & Compl & $19 \%$ & Yes \\
\hline Giovannini et al. & 2006 & Prosp & Liver & 92 & Compl & N/A & N/A \\
\hline Labgaa et al. & 2016 & Retro & Liver & 106 & Compl & N/A & Yes \\
\hline Hendifar et al. & 2016 & Retro & Pancreas & 106 & Compl & N/A & $N / A^{c}$ \\
\hline Ge et al. & 2017 & Retro & Colorectal & 626 & Compl & $15 \%$ & Yes \\
\hline Wierdak et al. & 2018 & Prosp & Colorectal & 105 & Infectious compl & $5 \mathrm{~g} / 1$ & N/A \\
\hline Wang et al. & 2018 & Retro & Colorectal & 193 & Major compl & $17 \%$ & Yes \\
\hline
\end{tabular}

Compl complications, $M V$ multivariable, N/A no answer, Prosp prospective, Retro retrospective, POPF postoperative pancreatic fistula

aultivariable analysis showing $\triangle \mathrm{Alb}$ to be an independent risk factor

${ }^{\mathrm{b}} \Delta$ Alb was not independently associated with postoperative complication

${ }^{\mathrm{C}}$ Found no association between $\Delta \mathrm{Alb}$ and complications but with $\Delta$ Alb and disease-free survival

(POD) 1 and 2 were higher in patients with major complications (Clavien grade $\geq \mathrm{III}$ ) than in patients without major complications. A threshold on POD 1 for relative $\triangle \mathrm{Alb}$ was found at $-27.3 \%$ from baseline.

In the context of emergency surgery, Kumar et al. measured pre- and postoperative serum albumin levels in 50 patients requiring exploratory laparotomy for organ perforation $(n=25)$, obstruction $(n=8)$, abdominal tuberculosis $(n=5)$, splenic injury $(n=4)$, stab wound $(n=$ $3)$, colon carcinoma $(n=3)$, and sigmoid volvulus $(n=2)$ (Kumar \& Sivakumar, 2020). The mean preoperative albumin value was $32.3 \pm 7.4 \mathrm{~g} / \mathrm{l}$ and the mean albumin value 4-6 hours after surgery was $27.5 \pm 7.9 \mathrm{~g} / \mathrm{l}$ (giving a mean drop of $4.8 \mathrm{~g} / \mathrm{l})$. The authors found that mean $\Delta$ Alb percentage was significantly associated with postoperative complications. They also mentioned specific complications, such as wound-related complications, acute respiratory distress syndrome, acute kidney injury, sepsis, anastomotic leak, or ileus. Wound dehiscence and leak were the most frequent complications (14\% each).

$\Delta \mathrm{Alb}$ was also shown to be predictive of complications after intestinal laparoscopic resection for Crohn's disease (Müller et al., 2018). In a retrospective of 182 patients, a relative $\Delta \mathrm{Alb}$ at a threshold of $24 \%$ was identified as an independent predictor of postoperative complications (HR 2.2, $p=0.04$ ).

\section{Esophageal surgery}

In a randomized controlled trial (RCT) comparing preoperative parenteral nutrition to oral nutrition before esophagectomy in 40 patients, Fan et al. found that, in the parenteral nutrition group, patients with weight gain and albumin drop had more pulmonary complications compared to patients with weight gain and albumin rise (Fan et al., 1989). Preoperative parenteral nutrition was not associated with a reduction of complications. No threshold for $\Delta \mathrm{Alb}$ was calculated and no multivariable analysis for postoperative complications assessing $\Delta \mathrm{Alb}$ was performed.

Recently, a multicentric (5 institutions) study in 1046 patients undergoing surgery for esophageal cancer identified $\triangle \mathrm{Alb}$ (calculated on POD 1) as an independent predictor of major complications (Labgaa et al., 2021). A $\Delta$ Alb cut-off of $11 \mathrm{~g} / \mathrm{L}$ was found as the best predictive threshold for complications. The entire cohort was separated into a training $(n=696)$ and validation $(n=350)$ cohort, both had consistent results. This study included respiratory complications and anastomotic leaks as specific complications.

\section{Gastric surgery}

Ai et al. found that in patients with normal preoperative albumin level and gastric cancer, $\Delta \mathrm{Alb}$ was associated with postoperative complications (OR 14, 95\% CI 6-32) 
(Ai et al., 2019). The authors found a discriminative threshold of relative $\triangle$ Alb on POD 1 at $19 \%$.

Liu et al. investigated in a retrospective study potential predictors of short-term complications after gastrectomy (Liu et al., 2017). Relative $\Delta$ Alb was the strongest predictive marker (OR 18, 95\% CI 6-53) with a discriminative threshold at 14\%, in comparison with CRP and lymphocytes.

\section{Liver surgery}

In a pilot study, Labgaa et al. found that maximum $\Delta \mathrm{Alb}$ also predicted postoperative complications after hepatectomy ( $n=106)$ (Labgaa et al., 2016). No specific $\Delta$ Alb threshold was evaluated.

A prospective study on 92 patients undergoing hepatectomy by Giovannini et al. found that absolute values of albumin (preoperatively and postoperatively) correlated with the occurrence of postoperative complications, similarly to $\Delta$ Alb but with a weaker correlation coefficient (Giovannini et al., 2006).

\section{Pancreas surgery}

Hendifar et al. investigated $\Delta \mathrm{Alb}$ as a prognostic factor in patients with resected pancreatic adenocarcinoma (Hendifar et al., 2016). They measured the preoperative serum albumin and dichotomized patients in two groups $(<3.5 \mathrm{~g} / \mathrm{dL}$ and $\geq 3.5 \mathrm{~g} / \mathrm{dL})$. Patients with albumin $<3.5 \mathrm{~g} /$ $\mathrm{dL}$ showed worse overall survival. In this study, $\Delta \mathrm{Alb}$ was not found as a predictive marker of postoperative complication.

\section{Colorectal surgery}

Ge et al. found in a retrospective study on 626 patients that albumin decrease $>15 \%$ within 2 postoperative days was independently associated with overall postoperative complications (Ge et al., 2017). Relative $\Delta$ Alb was also predictive of major complication, comprehensive complication index, LoS, and surgical site infections.

Wierdak et al. found in their prospective study in colorectal surgery that postoperative albumin decreased more intensively in patients with infectious complication(s) compared to patients without infectious complications (Wierdak et al., 2018). They found a $\Delta$ Alb threshold of $5 \mathrm{~g} / \mathrm{l}$. The main infectious complication was an anastomotic leak. Patients with postoperative complication(s) showed a significantly lower value of albumin on POD 2 and POD 3, compared to patients without complication.

In 2018, Wang et al. found in a retrospective study on 193 patients undergoing laparoscopic colorectal resection for cancer that $\Delta \mathrm{Alb}$ was an independent predictor of major complications (Wang et al., 2018). They found a threshold of $17 \%$ corresponding to an area under the curve of 0.916 .

\section{Quality and heterogeneity assessment}

The median Newcastle-Ottawa score of the included studies was 8 (IQR 7-8). All scores are summarized in Table 2. Sensitivity of $\Delta$ Alb to predict postoperative complications ranged from 63 to $84 \%$, whereas specificity ranged from 61 to $86 \%$ (positive and negative predictive values were not provided). A SROC plot is shown in Fig. 2.

\section{Discussion}

The present study provides a comprehensive overview on the predictive value of $\Delta \mathrm{Alb}$ for complications after GI surgery. Even though $\Delta \mathrm{Alb}$ thresholds were heterogeneous, $\Delta$ Alb was confirmed as a promising early predictor of morbidity.

Several articles that were not included focused on the predictive value of either preoperative albumin or absolute postoperative albumin level (Hiroi et al., 2019; Ryan et al., 2007). Conversely to single pre- or postoperative values of albumin, $\Delta$ Alb advantageously provides dynamic information on the surgical stress response and not only the nutritional status.

The pathophysiology of perioperative albumin metabolism still remains unclear. It has been suggested that the main reason for rapid albumin decrease postoperatively was due to the capillary leak induced by the inflammatory response to the surgical trauma (sequestration) (Mantziari et al., 2015). Other mechanisms that play a role in postoperative albumin drop are decrease of hepatic production and dilution of serum albumin (Mantziari et al., 2015). The latter is clearly dependent on the type of surgery and on fluid management. Concerning fluid management,

Table 2 Newcastle-Ottawa scores of all included articles $(n=16)$

\begin{tabular}{|c|c|c|c|c|c|}
\hline Authors & Year & Selection & Comparability & Outcome & Total \\
\hline Mantziari et al. & 2015 & 4 & 2 & 2 & 8 \\
\hline Hübner et al. & 2016 & 4 & 2 & 2 & 8 \\
\hline Labgaa et al. & 2017 & 4 & 2 & 2 & 8 \\
\hline Kumar et al. & 2020 & 4 & 0 & 2 & 6 \\
\hline Müller et al. & 2018 & 4 & 2 & 2 & 8 \\
\hline Galata et al. & 2020 & 4 & 2 & 2 & 8 \\
\hline Fan et al. & 1989 & 4 & 2 & 2 & 8 \\
\hline Labgaa et al. & 2020 & 4 & 2 & 2 & 8 \\
\hline Liu et al. & 2017 & 4 & 2 & 2 & 8 \\
\hline Ai et al. & 2019 & 4 & 2 & 2 & 8 \\
\hline Giovannini et al. & 2006 & 4 & 0 & 2 & 6 \\
\hline Labgaa et al. & 2016 & 4 & 2 & 2 & 8 \\
\hline Hendifar et al. & 2016 & 4 & 0 & 2 & 6 \\
\hline Ge et al. & 2017 & 4 & 2 & 2 & 8 \\
\hline Wierdak et al. & 2018 & 4 & 0 & 2 & 6 \\
\hline Wang et al. & 2018 & 4 & 2 & 2 & 8 \\
\hline
\end{tabular}




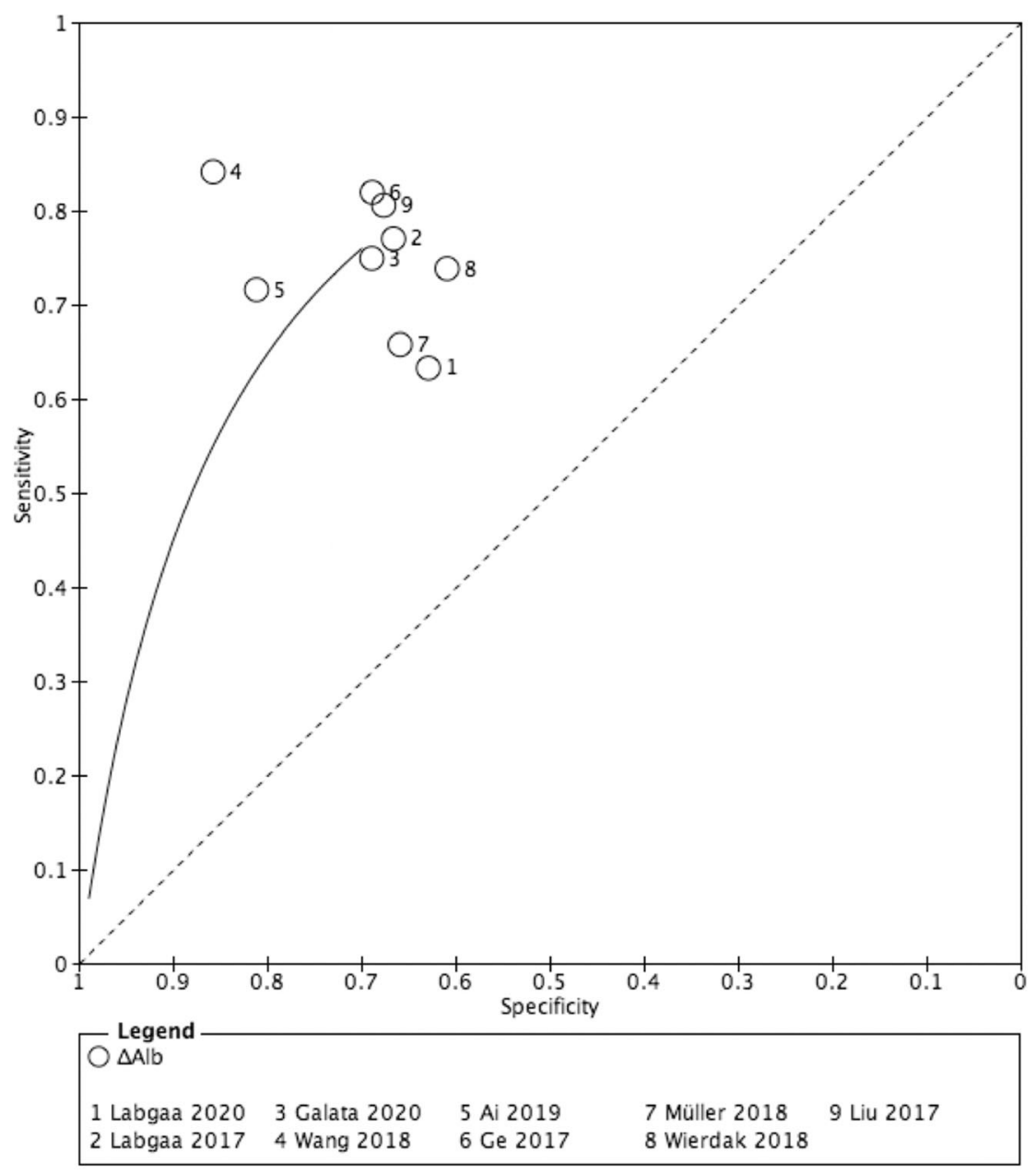

The 7 other studies were not included in the graph due to missing data regarding sensitivity and specificity.

Fig. 2 Summary plot of receiver operating characteristic (ROC) curves for $\Delta$ Alb

ERAS guidelines generally recommend goal-directed fluid therapy for the intraoperative phase and minimal intravenous fluids postoperatively, but these recommendations should be adapted for each specific surgery type. A recent study on major abdominal surgery found a significant correlation between fluid balance and weight change (additionally $\triangle \mathrm{Alb}$ correlated with fluid balance on POD 2). Furthermore, weight change was associated with longer LoS. Regarding laparoscopic and open colorectal surgery, major postoperative weight gain was shown to be associated with postoperative adverse outcomes (laparoscopy: respiratory complications and ileus, open: overall and respiratory complications). High volume of perioperative intravenous fluids after laparoscopic colic surgery was found to be an independent risk factor for overall, major, and respiratory complications. Normovolemia is usually therefore recommended postoperatively.

Regarding the kinetics, it has been shown that capillary leak after major surgery stops after POD 2 (Norberg et al., 2015). Moreover, several studies showed that the decrease of albumin mainly happened intraoperatively and during the first hours after major abdominal surgery (Komáromi et al., 2016; Norberg et al., 2016). After this rapid drop, serum albumin level remains stable for $72 \mathrm{~h}$ (Norberg et al., 2016). The kinetics of $\Delta \mathrm{Alb}$ is of interest, because it supports the use of $\Delta$ Alb as a very early marker of response to surgical stress (Fig. 3).

Future studies are needed to investigate two pivotal questions: (I) can we use this $\Delta \mathrm{Alb}$ to tailor postoperative surveillance; for example for early CT-scan or endoscopy 


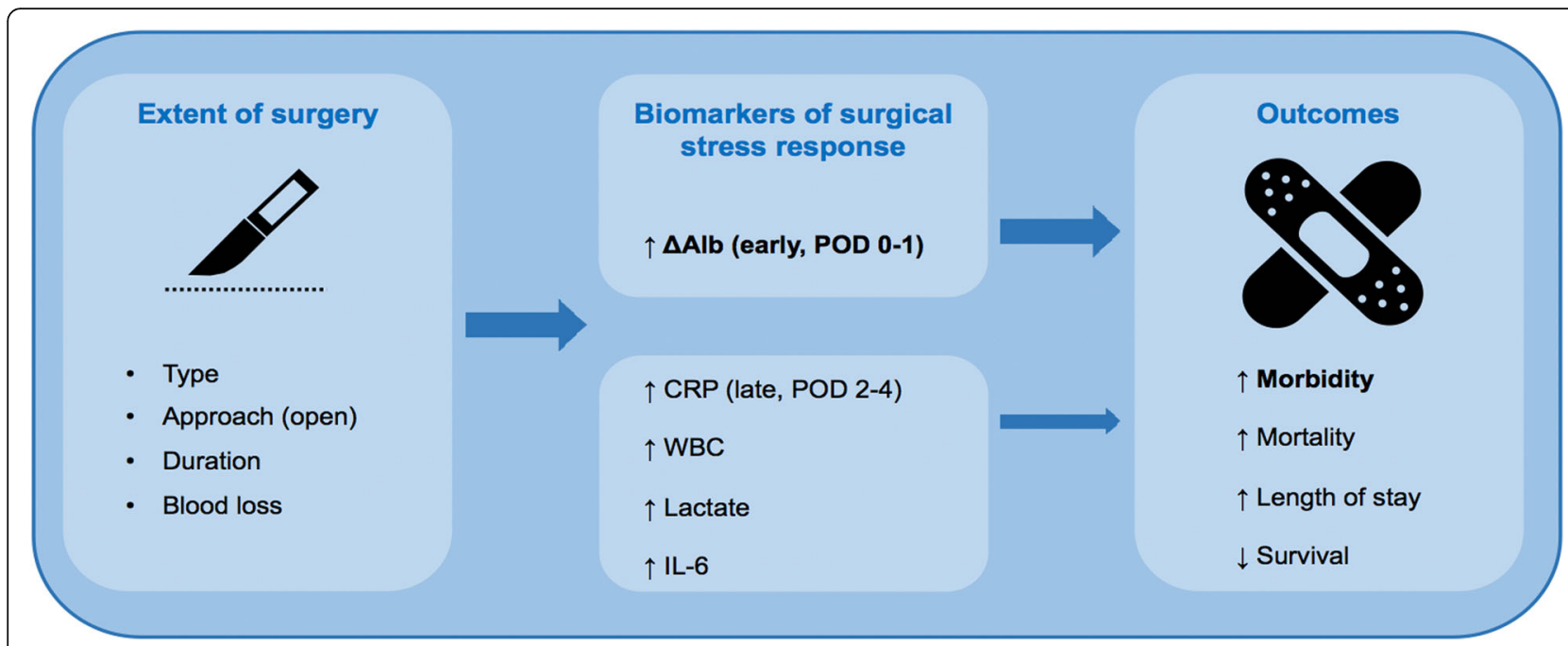

Fig. 3 Visual summary of $\Delta \mathrm{Alb}$ and other biomarkers and their relationship with extent of surgery and outcomes

and (II) is it possible to mitigate $\Delta \mathrm{Alb}$, for instance with albumin replacement, and would it improve outcomes? Despite the obvious clinical importance of the questions, answers would likely be speculative and clinical trials are thus needed to provide reliable data. Indeed, data regarding the necessity of perioperative albumin replacement in case of hypoalbuminemia remain scant and debated. Some studies showed that preoperative replacement with exogenous albumin in patients with hypoalbuminemia could decrease the rate of acute kidney injury (Lee et al., 2016). On the other hand, several studies did not find a positive effect of postoperative albumin replacement (MahkovicHergouth \& Kompan, 2011; Noonpradej \& Akaraborworn, 2020; Woods \& Kelley, 1993).

Additionally, $\Delta \mathrm{Alb}$ could also be used in combination with other markers in order to increase the predictive potential.

Some limitations of the present review need to be addressed. Due to the relative novelty of measuring $\triangle \mathrm{Alb}$, included studies had heterogeneous data and various types of GI surgeries were included. Therefore, the specificity of certain surgeries may play a role in the pathophysiology of $\Delta \mathrm{Alb}$. Nevertheless, $\Delta \mathrm{Alb}$ thresholds were constantly related to postoperative complications.

\section{Conclusion}

In summary, $\Delta \mathrm{Alb}$ is an interesting biomarker showing promising results, with the potential of better prediction of complications after GI surgery. Future studies are now needed to explore and understand the way to adapt perioperative management, in order to reduce postoperative complications.

\section{Abbreviations}

$\triangle$ Alb: Amplitude of albumin decrease; AMSTAR: Assessing the methodological quality of systematic reviews; CRP: C-reactive protein; Gl: Gastrointestinal; LOS: Length of stay; POD: Postoperative day; PRISMA: Preferred Reporting Items for Systematic Reviews and MetaAnalyses; RCT: Randomized controlled trial; SROC: Summary receiver operating characteristic

\section{Acknowledgements}

Not applicable.

\section{Authors' contributions}

Study concept and design: GRJ, MS, MH, ND, IL. Acquisition of data: GRJ, AS. Analysis and interpretation of data: GRJ, AS, MS, ND, MH, IL. Drafting of the manuscript: GRJ, AS, IL. Critical revision of the manuscript for important intellectual content: GRJ, AS, MS, ND, MH, IL. Final approval of the work to be published: GRJ, AS, MS, ND, MH, IL. Agreement to be accountable for all aspects of the work: GRJ, AS, MS, ND, MH, IL

\section{Funding}

This research did not receive any specific grant from funding agencies in the public, commercial, or not-for-profit sectors.

\section{Availability of data and materials}

The datasets used and/or analyzed during the current study are available from the corresponding author on reasonable request.

\section{Declarations}

Ethics approval and consent to participate

Not applicable.

Consent for publication

Not applicable.

Competing interests

The authors declare that they have no competing interests.

\section{Author details}

'Department of Visceral Surgery, Lausanne University Hospital (CHUV), University of Lausanne (UNIL), Rue du Bugnon 46, CH-1011 Lausanne, Switzerland. ${ }^{2}$ Graduate School of Health Sciences, University of Bern, Bern, Switzerland. ${ }^{3}$ Department of General and Visceral Surgery, HIB Hospital, Payerne, Switzerland. 
Received: 1 June 2021 Accepted: 5 January 2022

\section{Published online: 15 February 2022}

\section{References}

Ai S, Sun F, Liu Z, Yang Z, Wang J, Zhu Z, et al. Change in serum albumin level predicts short-term complications in patients with normal preoperative serum albumin after gastrectomy of gastric cancer. ANZ J Surg. 2019;89(7-8): E297-301. https://doi.org/10.1111/ans.15363.

Fan ST, Lau WY, Wong KK, Chan YP. Pre-operative parenteral nutrition in patients with oesophageal cancer: a prospective, randomised clinical trial. Clin Nutr. 1989;8(1):23-7. https://doi.org/10.1016/0261-5614(89)90021-6.

Galata C, Busse L, Birgin E, Weiß C, Hardt J, Reißfelder C, et al. Role of Albumin as a Nutritional and Prognostic Marker in Elective Intestinal Surgery. Can J Gastroenterol Hepatol. 2020;2020:7028216-8. https://doi.org/10.1155/2020/ 7028216.

Ge X, Dai X, Ding C, Tian H, Yang J, Gong J, et al. Early Postoperative Decrease of Serum Albumin Predicts Surgical Outcome in Patients Undergoing Colorectal Resection. Dis Colon Rectum. 2017;60(3):326-34. https://doi.org/10.1097/DCR. 0000000000000750 .

Giovannini I, Chiarla C, Giuliante F, Vellone M, Ardito F, Nuzzo G. The relationship between albumin, other plasma proteins and variables, and age in the acute phase response after liver resection in man. Amino Acids. 2006;31(4):463-9. https://doi.org/10.1007/s00726-005-0287-5.

Gonvers S, Jurt J, Joliat G-R, Halkic N, Melloul E, Hübner M, et al. Biological impact of an enhanced recovery after surgery programme in liver surgery. BJS Open. 2021;5(2):zraa015.

Hendifar A, Osipov A, Khanuja J, Nissen N, Naziri J, Yang W, et al. Influence of Body Mass Index and Albumin on Perioperative Morbidity and Clinical Outcomes in Resected Pancreatic Adenocarcinoma. PLoS One. 2016;11(3): e0152172. https://doi.org/10.1371/journal.pone.0152172.

Hiroi K, Matsusaki T, Kaku R, Umeda Y, Yagi T, Morimatsu H. Postoperative Course of Serum Albumin Levels and Organ Dysfunction After Liver Transplantation. Transplant Proc. 2019;51(8):2750-4. https://doi.org/10.1016/j.transproceed.201 9.01.199.

Hübner M, Mantziari S, Demartines N, Pralong F, Coti-Bertrand P, Schäfer M. Postoperative Albumin Drop Is a Marker for Surgical Stress and a Predictor for Clinical Outcome: A Pilot Study. Gastroenterol Res Pract. 2016;2016: 8743187-8. https://doi.org/10.1155/2016/8743187.

Jarnagin WR, Gonen M, Fong Y, DeMatteo RP, Ben-Porat L, Little S, et al. Improvement in perioperative outcome after hepatic resection: analysis of 1,803 consecutive cases over the past decade. Ann Surg. 2002;236(4):397406. https://doi.org/10.1097/00000658-200210000-00001.

Komáromi A, Estenberg U, Hammarqvist F, Rooyackers O, Wernerman J, Norberg $\AA$. Simultaneous assessment of the synthesis rate and transcapillary escape rate of albumin in inflammation and surgery. Crit Care. 2016;20(1):370. https://doi.org/10.1186/s13054-016-1536-6.

Kumar DM, Sivakumar S. Evaluation of the role of pre-operative albumin and its post-operative drop in the prediction of outcomes of emergency laparotomy. Int Surg J. 2020;7(4):1234-7. https://doi.org/10.18203/2349-2902. isj20201390.

Labgaa I, Demartines N, Hübner M. Biomarkers Capable to Early Predict Postoperative Complications: The Grail. Ann Surg. 2017a;266(6):e91-2. https:// doi.org/10.1097/SLA.0000000000001771.

Labgaa I, Demartines N, Hübner M. Serum Albumin: A Promising Biomarker to Anticipate Postoperative Complications. Dis Colon Rectum. 2017b;60(9):e616. https://doi.org/10.1097/DCR.0000000000000848.

Labgaa I, Joliat G-R, Demartines N, Hübner M. Serum albumin is an early predictor of complications after liver surgery. Dig Liver Dis. 2016;48(5):559-61. https://doi.org/10.1016/.j.dld.2016.01.004.

Labgaa I, Joliat G-R, Kefleyesus A, Mantziari S, Schäfer M, Demartines N, et al. Is postoperative decrease of serum albumin an early predictor of complications after major abdominal surgery? A prospective cohort study in a European centre. BMJ Open. 2017c;7(4):e013966. https://doi.org/10.1136/bmjopen-201 6-013966.

Labgaa I, Mantziari S, Genety M. Early Postoperative Decrease of Albumin is An Independent Predictor of Major Complications After Oncological Esophagectomy: A Multicenter Study. J Surg Oncol. 2021;123(2):462-9. https://doi.org/10.1002/jso.26317.

Lee E-H, Kim W-J, Kim J-Y, Chin J-H, Choi D-K, Sim J-Y, et al. Effect of Exogenous Albumin on the Incidence of Postoperative Acute Kidney Injury in Patients Undergoing Off-pump Coronary Artery Bypass Surgery with a Preoperative
Albumin Level of Less Than 4.0 g/dl. Anesthesiology. 2016;124(5):1001-11. https://doi.org/10.1097/ALN.0000000000001051.

Liu Z-J, Ge X-L, Ai S-C, Wang H-K, Sun F, Chen L, et al. Postoperative decrease of serum albumin predicts short-term complications in patients undergoing gastric cancer resection. World I Gastroenterol. 2017;23(27):4978-85. https:// doi.org/10.3748/wjg.v23.i27.4978.

Mahkovic-Hergouth K, Kompan L. Is replacement of albumin in major abdominal surgery useful? J Clin Anesth. 2011;23(1):42-6. https://doi.org/10.1016/j.jclina ne.2010.06.007.

Mantziari S, Hübner M, Coti-Bertrand P, Pralong F, Demartines N, Schäfer M. A Novel Approach to Major Surgery: Tracking Its Pathophysiologic Footprints. World J Surg. 2015;39(11):2641-51. https://doi.org/10.1007/s00268-015-31817.

McSorley ST, Khor BY, MacKay GJ, Horgan PG, McMillan DC. Examination of a CRP first approach for the detection of postoperative complications in patients undergoing surgery for colorectal cancer: A pragmatic study. Medicine. 2017; 96(7):e6133. https://doi.org/10.1097/MD.0000000000006133.

Müller C, Stift A, Argeny S, Bergmann M, Gnant M, Marolt S, et al. Delta albumin is a better prognostic marker for complications following laparoscopic intestinal resection for Crohn's disease than albumin alone - A retrospective cohort study. PLoS One. 2018;13(11):e0206911. https://doi.org/10.1371/journa I.pone.0206911.

Noonpradej S, Akaraborworn O. Intravenous Fluid of Choice in Major Abdominal Surgery: A Systematic Review. Crit Care Res Pract. 2020;2020:2170828-19. https://doi.org/10.1155/2020/2170828.

Norberg A, Rooyackers O, Segersvärd R, Wernerman J. Albumin Kinetics in Patients Undergoing Major Abdominal Surgery. PLoS One. 2015;10(8): e0136371. https://doi.org/10.1371/journal.pone.0136371.

Norberg Å, Rooyackers O, Segersvärd R, Wernerman J. Leakage of albumin in major abdominal surgery. Crit Care. 2016;20(1):113. https://doi.org/10.1186/ s13054-016-1283-8.

Ryan AM, Hearty A, Prichard RS, Cunningham A, Rowley SP, Reynolds JV. Association of Hypoalbuminemia on the First Postoperative Day and Complications Following Esophagectomy. J Gastrointest Surg. 2007;11(10): 1355-60. https://doi.org/10.1007/s11605-007-0223-y.

Straatman J, Cuesta MA, de Lange-de Klerk ESM, van der Peet DL. Hospital costanalysis of complications after major abdominal surgery. Dig Surg. 2015;32(2): 150-6. https://doi.org/10.1159/000371861.

Thorell A, Nygren J, Ljungqvist O. Insulin resistance: a marker of surgical stress. Curr Opin Clin Nutr Metab Care. 1999;2(1):69-78. https://doi.org/10.1097/ 00075197-199901000-00012

Vonlanthen R, Slankamenac K, Breitenstein S, Puhan MA, Muller MK, Hahnloser D, et al. The impact of complications on costs of major surgical procedures: a cost analysis of 1200 patients. Ann Surg. 2011;254(6):907-13. https://doi.org/1 $0.1097 /$ SLA.0b013e31821d4a43.

Wang Y, Wang H, Jiang J, Cao X, Liu Q. Early decrease in postoperative serum albumin predicts severe complications in patients with colorectal cancer after curative laparoscopic surgery. World J Surg Oncol. 2018;16(1):192. https://doi.org/10.1186/s12957-018-1493-4.

Warschkow R, Beutner U, Steffen T, Müller SA, Schmied BM, Güller U, et al. Safe and early discharge after colorectal surgery due to C-reactive protein: a diagnostic meta-analysis of 1832 patients. Ann Surg. 2012;256(2):245-50. https://doi.org/10.1097/SLA.0b013e31825b60fo.

Wierdak M, Pisarska M, Kuśnierz-Cabala B, Witowski J, Dworak J, Major P, et al. Changes in plasma albumin levels in early detection of infectious complications after laparoscopic colorectal cancer surgery with ERAS protocol. Surg Endosc. 2018;32(7):3225-33. https://doi.org/10.1007/s00464-01 8-6040-4.

Woods MS, Kelley H. Oncotic pressure, albumin and ileus: the effect of albumin replacement on postoperative ileus. Am Surg. 1993;59(11):758-63.

\section{Publisher's Note}

Springer Nature remains neutral with regard to jurisdictional claims in published maps and institutional affiliations. 